\title{
SURVEY ON LEGISLATION REGARDING WET COOLING SYSTEMS IN EUROPEAN COUNTRIES
}

\author{
K D Ricketts (katherine.ricketts@hpa.org.uk)¹, C Joseph ${ }^{1}$, J Lee², G Wewalka ${ }^{3}$, European Working Group for Legionella \\ Infections $^{1}$ \\ 1. Health Protection Agency, Centre for Infection, Respiratory Diseases Department, London, United Kingdom \\ 2. Health Protection Agency, Centre for Infection, Food Safety Microbiology Laboratory, London, United Kingdom \\ 3. Österreichische Agentur für Gesundheit und Ernährungssicherheit (AGES, Austrian Agency for Health and Food Safety), \\ Institute of Medical Microbiology and Hygiene, Vienna, Austria
}

Wet cooling systems are often associated with large outbreaks of Legionnaires' disease. Several European countries have legislation for registering such systems. The authors aimed to obtain an overview of the situation in Europe. A questionnaire survey was sent to 35 of the countries that collaborate in the European Working Group for Legionella Infections. In two countries it was passed to a regional level (to three regions in both Belgium and the United Kingdom), so that 39 countries or regions were sent the survey; 37 responded. Nine countries stated having legislation for the registration of wet cooling systems. Separate legislation exists at a regional level for two regions in Belgium and all three regions in the UK, giving a total of twelve countries/regions with legislation. In nine of these countries/regions, the legislation has been introduced since 2001. All of these countries/regions require periodic microbiological monitoring between twice a year and weekly; in nine, the legislation requires periodic inspection of the systems. Regulations for the registration of wet cooling systems should be required by public health authorities. During an outbreak of legionellosis, a register of wet cooling systems can speed up the investigation process considerably. The authors believe that the European Centre for Disease Prevention and Control (ECDC) should take the initiative to propose European Community $(E C)$ regulations for all Member States.

\section{Introduction}

Legionnaires' disease is an atypical pneumonic infection, acquired by inhaling aerosols containing Legionella spp. The legionella bacteria are commonly found in the natural and manmade aquatic environment, and enter the atmosphere through aerosol-generating outlets such as showers and cooling towers [1]. The first recognised outbreak of Legionnaires' disease occurred in 1976 at a hotel in Philadelphia [2] and was probably attributable to a cooling tower. Since then, wet cooling systems (including cooling towers, evaporative condensers and fluid coolers) have been established as some of the most common sources for outbreaks of legionellosis worldwide [1]. Wet cooling systems are heat rejection devices that utilise the evaporation of water to provide cooling. Common features are the recirculation of water which is sprayed or otherwise broken up into droplets in a counter current of air that is then ejected into the atmosphere. Some droplets may thus escape and form an aerosol outside of the cooling device. The recirculation of water can create good conditions for growth of legionellae.
Wet cooling systems can favour the growth of legionella by maintaining water temperatures of up to $35^{\circ} \mathrm{C}$ (temperatures in the range of $20^{\circ} \mathrm{C}$ to $45^{\circ} \mathrm{C}$ favour the growth of Legionella spp.) and by containing high levels of organic material and protozoa. About $2 \%$ of the water used in wet cooling systems escapes as aerosol and can drift more than 500 metres, in a few cases up to several kilometres, from its source $[3,4]$. When combined with poor maintenance and under-dosing of biocide, these systems can foster extensive growth of bacteria including Legionella pneumophila.

Every year the European Working Group for Legionella Infections (EWGLI) collects an aggregated dataset of all cases and outbreaks of Legionnaires' disease that have occurred in Europe during the previous year. Between 2002 and 2007, 44 outbreaks with cooling towers as the suspected source were reported in 11 countries, involving 1,175 cases (Table 1) [5-7].

For community-acquired outbreaks of Legionnaires' disease it is important to identify and treat the source as quickly as possible in order to prevent further infections. This can be a lengthy process if no register of wet cooling systems exists. Several European countries, especially those which have already experienced large cooling tower outbreaks, are known to have legislation for registering such devices. To obtain an overview of the situation in Europe, the authors conducted a questionnaire survey among the countries that participate in EWGLI.

\section{Methods}

A questionnaire was approved by the steering committee for the European Surveillance Scheme for Travel Associated Legionnaires' disease (EWGLINET) and sent to 35 EWGLI collaborating countries; it was passed to a regional level in Belgium (Brussels, Flanders and Wallonie) and the UK (England and Wales, Northern Ireland and Scotland). Therefore, 39 countries or regions were asked to participate.

The questionnaire included the following questions, and allowed space for further comments:

- Does your country have legislation for registering wet cooling systems?

- If yes, is the legislation national or regional?

- Which ministry issued the legislation?

- In what year was the legislation introduced? 
- Is there an official requirement for periodical inspection of wet cooling systems?

- Who is responsible for the periodic inspection of wet cooling systems?

- Is there an official requirement for microbiological monitoring?

- Are there penalties imposed for unregistered wet cooling systems?

- Does a register of wet cooling systems exist?

- Who holds the register?

- How does the authority get the information?

- Who is responsible for maintaining the information?

The initial results were presented at the 22nd EWGLI conference in Stockholm [8], and comment and interpretation was sought from the collaborating countries.

\section{Results}

Representatives from 37 collaborating countries or regions (94.9\%) returned the questionnaire. Of these, 12 (32.4\%) reported having legislation requiring the registration of wet cooling systems at a national level (Andorra, France, Malta, The Netherlands, Norway and Spain) or a regional level (Belgium: Wallonie and Flanders; UK: England and Wales, Northern Ireland and Scotland; and the Russian Federation) (Table 2). The countries or regions that returned the questionnaire and do not have such legislation are: Austria, Belgium (Brussels), Bulgaria, Croatia, Czech Republic, Denmark, Estonia, Finland, Germany, Greece, Hungary, Ireland, Israel, Italy, Latvia, Lithuania, Luxembourg, Poland, Portugal, Romania, Slovak Republic, Slovenia, Sweden, Switzerland, Turkey.

In five countries or regions this legislation is issued by the Ministry of Public Health, in three by the Ministry or Department of the Environment, in two by the Ministry or Department of Trade and Industry, and in one by the Department of Industrial Construction.
In the Netherlands, the registration is voluntary and is covered by environmental legislation; it is anticipated that legislation requiring the registration of new wet cooling systems will be introduced in 2009. In England and Wales, Scotland and Northern Ireland, legislation has existed since 1992 or 1994; in all other countries or regions the legislation has been introduced since 2001 .

All countries or regions which have legislation require periodic microbiological monitoring between twice a year and weekly; 'microbiological monitoring' was not further specified in the questionnaire and the responses are likely to refer to dipstick tests rather than to full environmental sampling. In nine countries the legislation requires periodic inspection of the systems. In all twelve of the countries or regions which have legislation, a register of wet cooling systems exists. This register is held by national authorities $(n=2)$, regional authorities $(n=3)$ or local authorities $(n=7)$, and in nine of these countries/regions, penalties are imposed for unregistered systems. In eight of the nine countries/regions where penalties can be imposed, the owner of the system is responsible for ensuring that the information on the register is correct.

Of the 25 (67.6\%) countries or regions with no legislation for registering wet cooling systems (Table 3 ), five require microbiological monitoring and four stated that technical standards require periodic inspections; two will impose penalties for not following these

T A B L E 3

Countries or regions without legislation on the registration of wet cooling systems, EWGLI survey, 2007

\begin{tabular}{|c|c|c|c|c|}
\hline $\begin{array}{c}\text { Number of } \\
\text { countries or } \\
\text { regions }\end{array}$ & $\begin{array}{c}\text { Periodical } \\
\text { inspections }\end{array}$ & $\begin{array}{c}\text { Microbiological } \\
\text { monitoring }\end{array}$ & $\begin{array}{c}\text { Does } \\
\text { register } \\
\text { exist }\end{array}$ & $\begin{array}{c}\text { Who holds } \\
\text { register } \\
\text { (authority) }\end{array}$ \\
\hline 25 & $\begin{array}{c}21 \text { no } \\
4 \text { yes }\end{array}$ & $\begin{array}{c}20 \text { no } \\
5 \text { yes }\end{array}$ & $\begin{array}{c}23 \text { no } \\
2 \text { yes }\end{array}$ & $\begin{array}{c}1 \text { national } \\
1 \text { regional }\end{array}$ \\
\hline
\end{tabular}

T A B L E 1

Outbreaks of Legionnaires' disease with wet cooling systems as the suspected source, as reported to the EWGLI annual dataset by collaborating countries, 2002-2007 ( $\mathrm{n}=44$ outbreaks)

\begin{tabular}{|c|c|c|c|c|c|c|c|c|c|c|c|c|}
\hline \multirow{2}{*}{$\begin{array}{l}\text { Country } \\
\text { (region) of } \\
\text { outbreak }\end{array}$} & \multicolumn{2}{|c|}{2002} & \multicolumn{2}{|c|}{2003} & \multicolumn{2}{|c|}{2004} & \multicolumn{2}{|c|}{2005} & \multicolumn{2}{|c|}{2006} & \multicolumn{2}{|c|}{2007} \\
\hline & $\begin{array}{c}\text { WCS } \\
\text { outbreaks }\end{array}$ & $\begin{array}{l}\text { Number } \\
\text { of cases }\end{array}$ & $\begin{array}{c}\text { WCS } \\
\text { outbreaks }\end{array}$ & $\begin{array}{l}\text { Number } \\
\text { of cases }\end{array}$ & $\begin{array}{c}\text { WCS } \\
\text { outbreaks }\end{array}$ & $\begin{array}{l}\text { Number } \\
\text { of cases }\end{array}$ & $\begin{array}{c}\text { WCS } \\
\text { outbreaks }\end{array}$ & $\begin{array}{l}\text { Number } \\
\text { of cases }\end{array}$ & $\begin{array}{c}\text { WCS } \\
\text { outbreaks }\end{array}$ & $\begin{array}{l}\text { Number } \\
\text { of cases }\end{array}$ & $\begin{array}{c}\text { WCS } \\
\text { outbreaks }\end{array}$ & $\begin{array}{l}\text { Number } \\
\text { of cases }\end{array}$ \\
\hline Austria & & & & & & & & & & & 1 & 9 \\
\hline France & 2 & $22 ; 31$ & 3 & $31 ; 24 ; 84$ & & & 1 & 34 & 3 & $29 ; 10 ; 12$ & 1 & 9 \\
\hline Italy & & & 1 & 15 & & & & & & & & \\
\hline Netherlands & & & & & & & & & 3 & $31 ; 9 ; 10$ & & \\
\hline Norway & 1 & 28 & & & & & 1 & 58 & & & & \\
\hline Portgual & & & & & & & & & 2 & $3 ; 21$ & & \\
\hline Spain & 2 & $108 ; 9$ & 4 & $11^{\star \star} ; 6 ; 13 ; 6$ & 2 & $32 ; 29$ & 4 & $12 ; 15 ; 50 ; 4$ & 1 & 146 & 1 & 18 \\
\hline Sweden & & & & & 1 & 32 & & & & & & \\
\hline $\begin{array}{l}\text { UK (England } \\
\text { and Wales) }\end{array}$ & 2 & $6 ; 146$ & 1 & 27 & 1 & 4 & 2 & $3 ; 2$ & & & & \\
\hline $\begin{array}{l}\text { UK (Northern } \\
\text { Ireland) }\end{array}$ & 1 & 3 & & & & & 1 & 3 & & & & \\
\hline UK (Scotland) & & & & & 1 & 7 & 1 & $3^{*}$ & & & & \\
\hline
\end{tabular}

WCS outbreaks $=$ wet cooling system outbreaks

* Two Scottish cases and one English case

** Associated with an evaporative condenser

NB: These figures were provisional reports at the time of submission to EWGLI; subsequent reports may cite different case numbers. Some countries (Norway, Spain and Sweden) have provided updated data to reflect final case numbers for these outbreaks. 
T A B L E 2

\begin{tabular}{|c|c|c|c|c|c|c|c|c|c|c|c|c|}
\hline 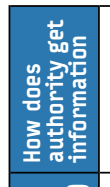 & 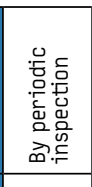 & 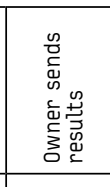 & 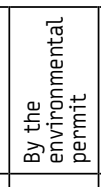 & 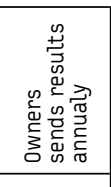 & 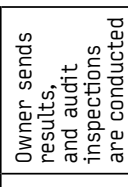 & 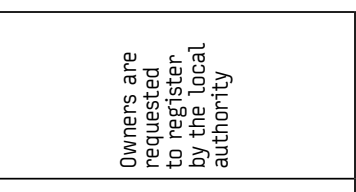 & 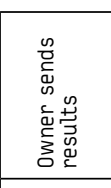 & 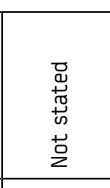 & 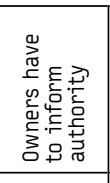 & 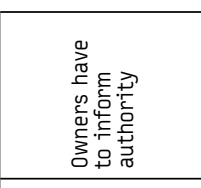 & 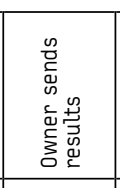 & 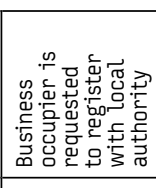 \\
\hline 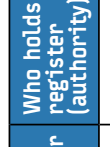 & 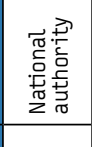 & 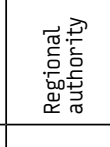 & & 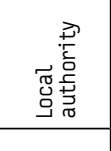 & 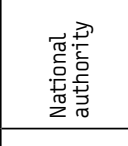 & 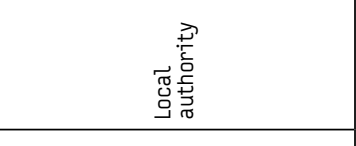 & 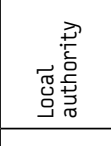 & 居 & 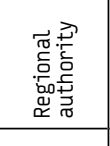 & 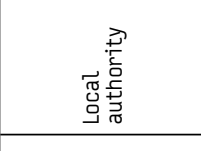 & 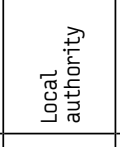 & 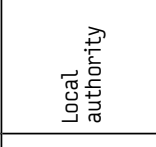 \\
\hline 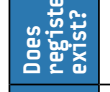 & 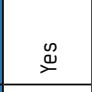 & 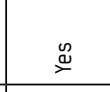 & $\frac{0}{2}$ & $\stackrel{\mathscr{g}}{\underline{\underline{m}}}$ & 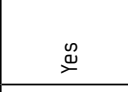 & 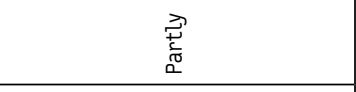 & $\stackrel{\underline{\underline{w}}}{\underline{\underline{\omega}}}$ & 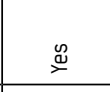 & 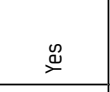 & 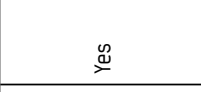 & 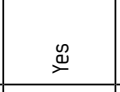 & 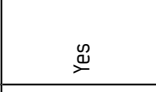 \\
\hline 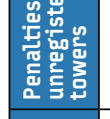 & $\stackrel{\infty}{ٍ}$ & 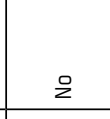 & 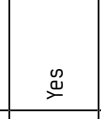 & 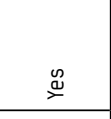 & 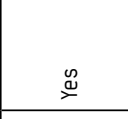 & $i$ & $\stackrel{\mathscr{\infty}}{ٍ}$ & $\begin{array}{l}\text { 言 } \\
\text { 产 } \\
\text { 营 } \\
\end{array}$ & 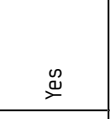 & $\stackrel{\mathscr{\omega}}{\underline{\underline{\underline{m}}}}$ & 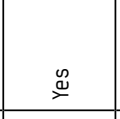 & 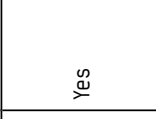 \\
\hline $\begin{array}{l}\frac{a}{0} \\
\frac{a}{2} \\
\frac{a}{2}\end{array}$ & 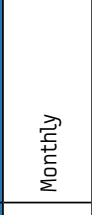 & 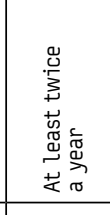 & 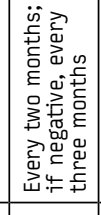 & 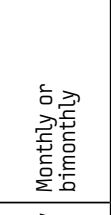 & 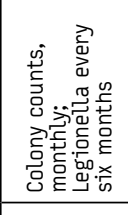 & 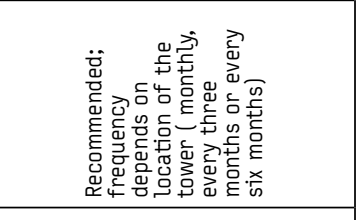 & 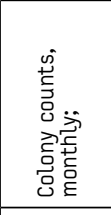 & 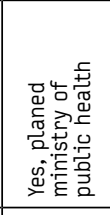 & 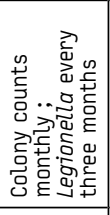 & 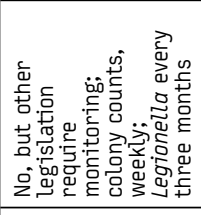 & 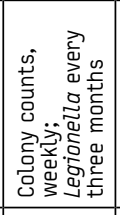 & 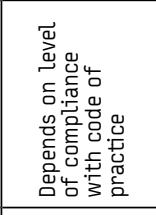 \\
\hline 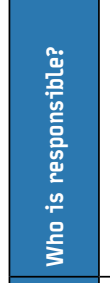 & 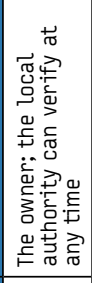 & 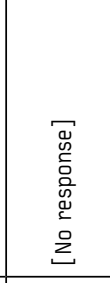 & 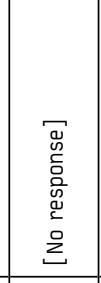 & 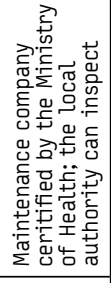 & 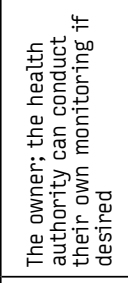 & 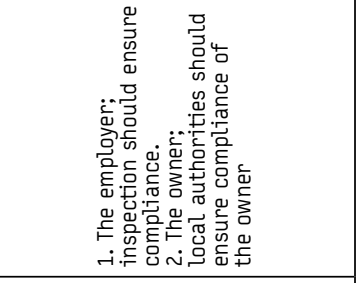 & 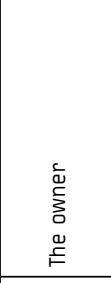 & 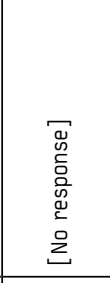 & 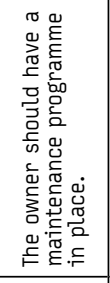 & 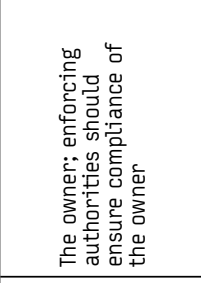 & 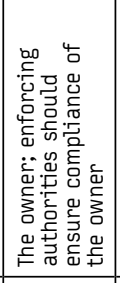 & 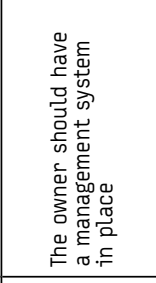 \\
\hline 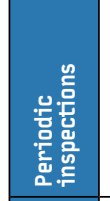 & 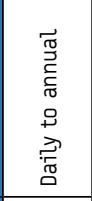 & $\frac{0}{2}$ & 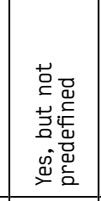 & 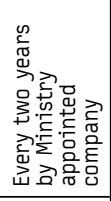 & 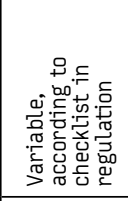 & 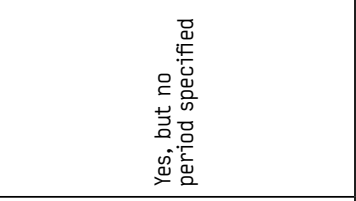 & 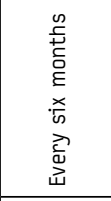 & $\frac{0}{2}$ & 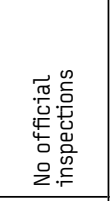 & 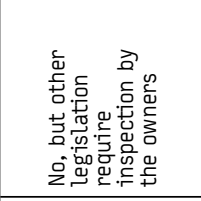 & 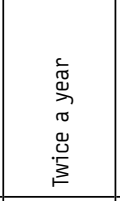 & 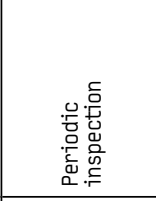 \\
\hline 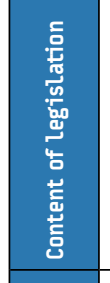 & 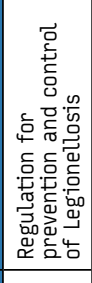 & 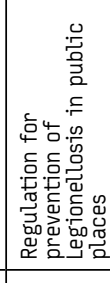 & 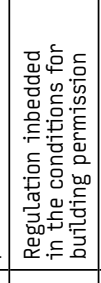 & 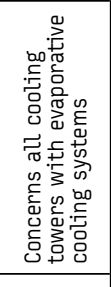 & 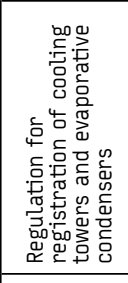 & 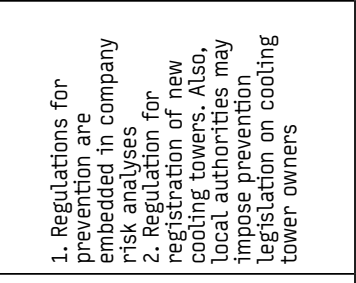 & 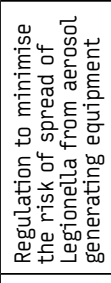 & 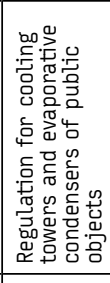 & 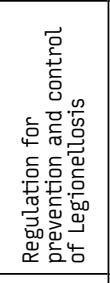 & 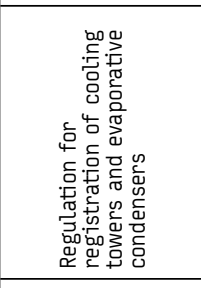 & 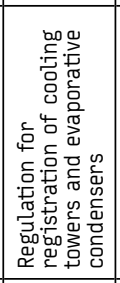 & 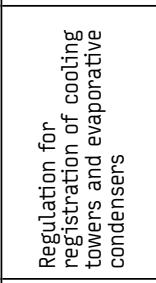 \\
\hline 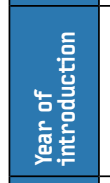 & 芯 & 总 & 总 & 总 & 总 & 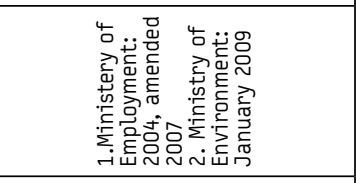 & 总 & 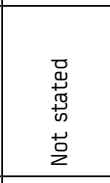 & 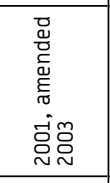 & 鷤 & 莺 & $\stackrel{\widetilde{g}}{g}$ \\
\hline 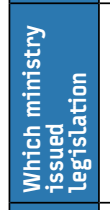 & 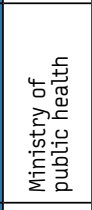 & 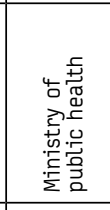 & 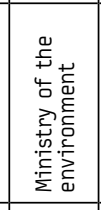 & 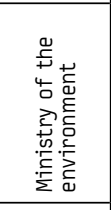 & 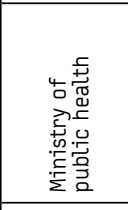 & 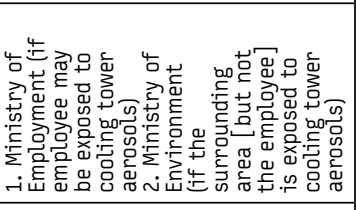 & 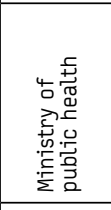 & 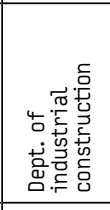 & 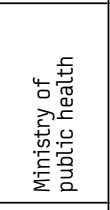 & 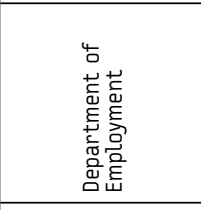 & 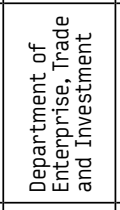 & 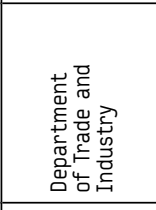 \\
\hline 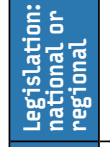 & 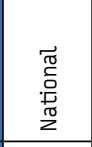 & 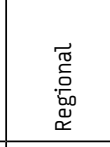 & 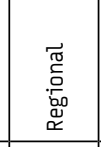 & 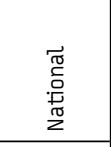 & \begin{tabular}{l} 
总 \\
产 \\
\multirow{2}{*}{} \\
\end{tabular} & 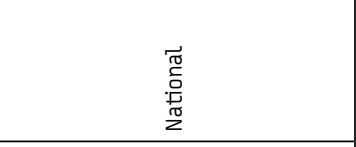 & 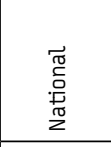 & 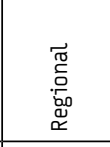 & 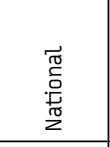 & 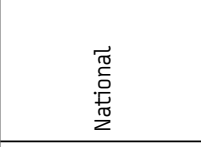 & 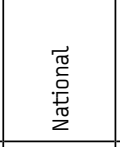 & 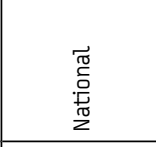 \\
\hline 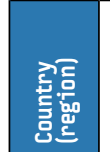 & $\begin{array}{l}\text { 畜 } \\
\text { 毫 }\end{array}$ & 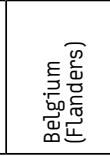 & 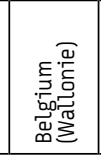 & 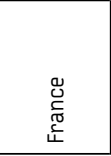 & 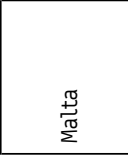 & 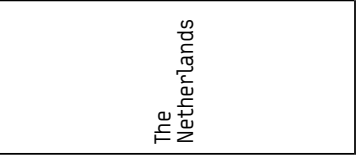 & $\begin{array}{l}\text { 恋 } \\
\text { 竞 }\end{array}$ & 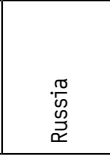 & 畜 & 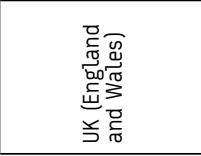 & 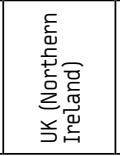 & 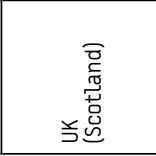 \\
\hline
\end{tabular}


standards. Of these 25 countries or regions, only one country (Luxembourg) and one region (Brussels) have a register of wet cooling systems, and because Brussels' register includes only new systems, it is not comprehensive.

\section{Discussion}

Minimising the number of cases of legionellosis caused by wet cooling systems should be an important target for public health authorities1. A preliminary risk assessment by Ambroise et al. [9] showed that exposure through cooling towers led to more cases of Legionnaires' disease (by a factor of 100-130) than exposure during showering, whilst Lock et al. detailed the high cost of an outbreak of Legionnaires' disease caused by a cooling tower [10]. The EWGLI annual dataset (Table 1) shows that between 2002 and 2007 there were an average of 7.3 outbreaks caused by wet cooling systems each year, involving 1,175 cases (an average of 195.8 cases per year and 26.7 per outbreak). In comparison, 215 outbreaks (35.8 per year) with 784 cases were associated with water systems (an average of 130.7 cases per year and 3.6 per outbreak) $[6,7]$. It should be noted that a large number of outbreaks are never properly attributed to sources [7], and that the larger ones (often associated with wet cooling systems) are more likely to be attributed to a source than smaller outbreaks $[3,11,12]$.

In most of the countries or regions that have regulations for the registration of wet cooling systems, these were introduced following the recognition of outbreaks caused by such devices. Regulations were introduced in England, Wales and Scotland in 1992 [13] following Public Enquiries resulting from the Stafford hospital outbreak [14] and the BBC outbreak [15], both of which were caused by cooling towers. After a big outbreak in a town near Madrid in 1997 [16], the first regional law was issued in Spain. This was followed by laws in many other regions of Spain and by a national law in 2001 (later revised in 2003). In France a number of outbreaks, including the 2003 outbreak in Lens [3], led to specific regulations in 2004; in Norway regulations to minimise the risk of spread of legionella from aerosolizing equipment followed an outbreak caused by an air washer [4]. In the Netherlands a cooling tower related outbreak in Amsterdam in 2006 [17] was the impetus for the introduction of specific rules.

Of those eleven countries or regions that experienced wet cooling system outbreaks which were reported to EWGLI between 2002 and 2007 (Table 1), three reported having no legislation for registering wet cooling systems (Italy, Portugal and Sweden). However, the three countries or regions that have reported the most outbreaks over this period (Spain, France and England and Wales) all have legislation. These three countries or regions require frequent microbiological monitoring, keep a register of towers and impose penalties for unregistered systems. The only area where they may have less rigid legislation than countries or regions with fewer outbreaks is in regards to periodic monitoring. Spain suffers from the highest number of outbreaks and does not require periodic official inspection of systems, but there are different levels of response following positive Legionella spp. counts depending upon how infected the system is. France only requires inspections every two years, and England and Wales do not have a set frequency for inspections by local authorities (however the obligation to monitor rests with the wet cooling system owners and the enforcing authorities should ensure that they fulfil this obligation) [18].
It is difficult to draw solid conclusions from this data because there are many differences in ascertainment, data collection, and reporting systems between countries. Nevertheless, there is enough evidence to suggest that developing water safety plans for wet cooling systems, including system assessment, monitoring and management, is the preferred approach for managing the health risks associated with exposure to Legionella spp. [19,20]. Specific legislation is needed to ensure that authorities responsible for the safety of water systems or buildings develop and follow water safety plans. Most outbreaks associated with wet cooling systems are preventable, and such legislation could therefore lead to a substantial reduction in morbidity and mortality from Legionnaires' disease.

Regulations for the registration of wet cooling systems should also be required by health systems. During an outbreak of legionellosis, identifying and containing the source as quickly as possible should be one of the initial aims of an outbreak control team. In order to achieve this, improving surveillance to ensure the rapid detection of cases and clusters is important, but a register of wet cooling systems can also be an invaluable starting point and speed up the process considerably [21]. At present only 12 European countries or regions have specific legislation for this. Several EWGLI collaborating countries that do not currently have such legislation have suggested that European Community (EC) regulations for the registration of wet cooling systems and the prevention of legionellosis are required, and that the European Centre for Disease Prevention and Control (ECDC) should take the initiative to propose such regulations.

\section{Acknowledgements}

List of responding EWGLINET collaborating centres: Andorra, Austria, Belgium (Wallonie, Brussels Capital and Flanders), Bulgaria, Croatia, Czech Republic, Denmark, Estonia, Finland, France, Germany, Greece, Hungary, Ireland, Israel, Italy, Latvia, Lithuania, Luxembourg, Malta, The Netherlands, Norway, Poland, Portugal, Romania, Russia, Slovak Republic, Slovenia, Spain, Sweden, Switzerland, Turkey, UK (England and Wales, Northern Ireland and Scotland)

Supporting members of EWGLINET steering committee: Dr B Brodhun, Ms C Campese, Dr R Cano, Dr V Drasar, Dr C Rota, Dr I Tomova, Dr S Uldum, Dr J van Wijingaarden, and Dr J Takkinen.

\section{References}

1. Bartram J, Chartier Y, Lee, JV, Pond K, Surman-Lee S, editors. Legionella and the prevention of legionellosis. Eds: Geneva: World Health Organization; 2006. Available from: http://www.who.int/water_sanitation_health/emerging/ legionella.pdf

2. Fraser DW, Tsai TR, Orenstein W, Parkin WE, Beecham HJ, Sharrar RG, et al. Legionnaires' disease: description of an epidemic of pneumonia. N Engl J Med. 1977;297(22):1189-97.

3. Nguyen TM, Ilef D, Jarraud S, Rouil L, Campese C, Che D, et al. A communitywide outbreak of Legionnaires' disease linked to industrial cooling towers-how far can contaminated aerosols spread? J Infect Dis. 2006;193(1):102-11.

4. Nygård K, Werner-Johansen $\emptyset$, Rønsen S, Caugant DA, Simonsen $\emptyset$, Kanestrøm $A$, et al. An outbreak of Legionnaires disease' caused by long-distance spread from an industrial air scrubber in Sarpsborg, Norway. Clin Infect Dis. 2008;46(1):61-9.

5. Joseph CA, European Working Group for Legionella Infections. Legionnaires' disease in Europe 2000-2002. Epidemiol Infect. 2004;132(3):417-24.

6. Ricketts KD, Joseph C. Legionnaires' disease in Europe 2003-2004. Euro Surveill. 2005;10(12):pii=588. Available from: http://www.eurosurveillance. org/ViewArticle.aspx?ArticleId=588. 
7. Ricketts KD, Joseph CA. Legionnaires' disease in Europe: 2005-2006. Euro Surveill. 2007;12(12):pii=753. Available from: http://www.eurosurveillance. org/ViewArticle. aspx?ArticleId=753.

8. Wewalka G, Ricketts K. Survey on legislation about evaporative cooling towers in European countries. Proceedings of 22nd meeting of the European Working Group for Legionella Infections, Stockholm, Sweden; 2007. p. 77.

9. Ambroise D, Harteman P. Influence of variability in measuring airborne bacteria during the evaluation of the risk of infection. Int J Hyg Environ Health (in press).

10. Lock K, Millett C, Heathcock R, Joseph CA, Harrison TG, Lee JV, et al. on behalf of the Outbreak Control Team. Public health and economic costs of investigating a suspected outbreak of Legionnaires' disease. Epidemiol Infect. 2008;136(10):1306-1314.

11. García-Fulgueiras A, Navarro C, Fenoll D, García J, González-Diego P, JiménezBuñuales T, et al. Legionnaires' disease outbreak in Murcia, Spain. Emerg Infect Dis. 2003;9(8):915-21.

12. Legionella section. Legionnaires' disease in residents of England and Wales: 2006. Health Protection Report Vol 2 No 5, 1 February 2008. Available from: http://www.hpa.org.uk/hpr/archives/2008/hpr0508.pdf.

13. Health and Safety Commission. The Notification of Cooling Towers and Evaporative Condensers Regulations 1992. The prevention or control of legionellosis (including Legionnaires' disease): approved code of practice. London: HMSO, 1991.

14. Committee of Inquiry. First report of the Committee of Inquiry into the outbreak of Legionnaires' disease in Stafford in April 1985.

15. Cunningham D, editor. Broadcasting House Legionnaires' disease. Report of the Westminster Action Committee. London: Department of Environmental Services, Westminster Council House; 1988.

16. Anonymous. Brote de Neumonía por Legionella de Alcalá de Henares”. Bol Ep Cm. Vol. 5. No 4. Abril 1997.

17. Hoek JAR van den, Ijzerman EPF, Coutinho RA. Legionella-uitbraak in Amsterdam: koeltoren als bron. Ned Tijdschr Geneeskd. 2006;150:1808-11.

18. Health and Safety Commission. Approved Code of Practice on the prevention and control of legionellosis (including Legionnaires' disease) (L8) (2002)

19. World Health Organization. Guidelines for drinking water quality, $3 r d$ ed. Vol 1. Recommendations, Geneva: World Health Organization; 2004. Available from: http://www.who.int/water_sanitation_health/dwq/gdwq3rev/en/

20. Davison A, Howard G, Stevens M, Callan P, Fewtrell L, Deere D, et al. Water safety plans: managing drinking water quality from catchment to consumer Geneva: World Health Organization; 2005. Available from: http://www.who.int/ water_sanitation_health/dwq/wsp0506/en/

21. Kirrage D, Reynolds G, Smith GE, Olowokure B; Hereford Legionnaires Outbreak Control Team. Investigation of an outbreak of Legionnaires' disease: Hereford, UK 2003. Respir Med. 2007;101(8):1639-44.

This article was published on 18 September 2008.

Citation style for this article: Ricketts KD, Joseph C, Lee J, Wewalka G, European Working in European countries. Euro Surveill. 2008;13(38):pii=18982. Available online: http:// in European countries. Euro Surveill. 2008;13(38):pij=18982. 